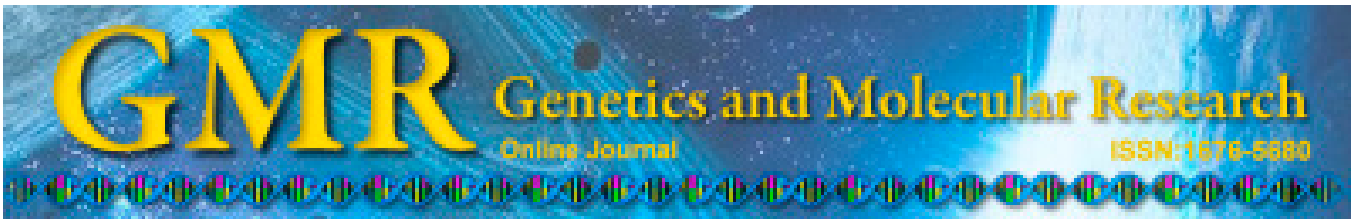

\title{
Predictive value of CK20 in evaluating the efficacy of treatment and prognosis after surgery for colorectal cancer
}

\author{
W.X. Li ${ }^{1 *}$, H.W. Xiao ${ }^{2 *}$, X.Q. Hong ${ }^{3}$ and W.X. Niu ${ }^{4}$ \\ ${ }^{1}$ Department of General Surgery, Zhongshan Hospital, Fudan University, \\ Shanghai, China \\ ${ }^{2}$ Department of Surgery, Jinshan Tinglin Hospital, Shanghai, China \\ ${ }^{3}$ Department of General Surgery, Zhongshan Hospital, Fudan University, \\ Shanghai, China \\ ${ }^{4}$ Department of General Surgery, Zhongshan Hospital, Fudan University, \\ Shanghai, China \\ *These authors contributed equally to this study. \\ Corresponding author: W.X. Niu \\ E-mail: NIUwx021@126.com
}

Genet. Mol. Res. 14 (2): 5823-5829 (2015)

Received November 13, 2014

Accepted February 9, 2015

Published May 29, 2015

DOI http://dx.doi.org/10.4238/2015.May.29.14

\begin{abstract}
Herein, correlations between expression levels of CK20 and efficacy of treatment and postoperative prognosis of colorectal cancer were evaluated to elucidate the clinical value of CK20. Postoperative follow-up was performed on 62 patients who underwent surgery for colorectal cancer between January 2010 and December 2010. Samples of tumor tissues and intraperitoneal drainage fluids were collected. Blood samples were obtained during the 2-year follow-up period. The expression of CK20 in surgical specimen, intraperitoneal drainage fluids, and postoperative serum samples was quantified by enzyme-linked immunosorbent assay, RT-PCR, and western blotting. Correlation between the levels of CK20 and postoperative outcomes was investigated by Spearman correlation analysis. In both tumor
\end{abstract}


specimens and intraperitoneal drainage fluids, CK20 levels were lower in patients with earlier cancer stages than in those at later stages. During postoperative follow-up, serum negative CK20 patients had significantly higher 3-year survival rates than serum positive CK20 patients. All differences were statistically significant $(\mathrm{P}<0.05)$. CK20 levels can provide clinically valuable information on the postoperative prognosis of patients with colorectal cancer.

Key words: CK20 expression; Lymph node; Colorectal cancer; Prognosis

\section{INTRODUCTION}

Colorectal cancer is a common malignant tumor with a 3-year survival rate of about $70 \%$ after surgical resection (Zhang et al., 2008; Bertz et al., 2014). Although modern cancer therapy can effectively control progression of colorectal carcinoma, there is a trend towards increased mortality (Montezuma et al., 2013). Current techniques for the evaluation of colorectal cancer include pathological examination, tumor marker level measurement, and observation of clinical symptoms, although they are all associated with inherent limitations that leads to difficulty in detecting early stage tumors and lymph node metastases (Eisenberg-Lerner et al., 2009; Littman and Rudensky, 2010; Pavlidis and Pentheroudakis, 2012). Therefore, a novel method for effectively predicting postoperative prognosis of colorectal cancer is critical for the management of tumor progression and metastasis. This study focused on the potential role of CK20 in evaluating the efficacy of treatment and postoperative prognosis of colorectal cancer.

\section{MATERIAL AND METHODS}

\section{Clinical information}

The protocol of this study was approved by the Ethics Committee of Zhongshan Hospital, Fudan University (Shanghai, China). Informed consent was obtained from all subjects. A total of 62 patients undergoing surgical resection for colorectal cancer at Zhongshan Hospital, Fudan University between January 2010 and December 2010 were included in the study. There were 41 men and 21 women, aged between 52 and 79 years (mean $63.4 \pm 5.2$ years). All patients were diagnosed with colorectal cancer by clinical examination and computed tomography (Takata et al., 2014), underwent surgical resection of colorectal tumors, and had no mental disease or family history of mental disorders. All patients had normal cognitive function and had no disease that could affect the results of the study. Exclusion criteria included: 1) severe adverse events during the follow-up period; 2) violation of standard treatment protocol; and 3) withdrawal from the study during the follow-up period (von Freyend et al., 2011).

\section{Reagents}

The enzyme-linked immunosorbent assay (ELISA) kit was from Bogoo Biotech (China). Primers used in RT-PCR were purchased from Agilent Stratagene (USA). An ultra- 
violet spectrophotometer (Model UV1901PC) was provided by Aucy Instruments (China). Anti-CK20 antibody was purchased from CST (USA). Other reagents including EDTA retrieval buffer, electrophoresis buffer, and elution buffer were prepared in-house. Primers for CK20 were: forward, 5'-CGCAC CTCCC AGAGC CTTGA-3'; reverse, 5'-ATTCG TTGTT CGGGC GTTCC-3'; expected length: 187 bp.

\section{Sample collection and assays}

Colorectal tumor samples from surgical resections and intraperitoneal drainage fluid samples at 7 days after surgery were collected for western blotting, RT-PCR, and ELISA to quantify the level of expression of CK20. All patients underwent postoperative follow-up. During the first 2 years, follow-up was performed every 2 months. After 2 years, follow-up was performed every 6 months. The last follow-up was in December 2013. At each follow-up, $10 \mathrm{~mL}$ venous blood was collected from the external elbow and centrifuged to obtain serum. CK20 levels in serum were further assayed by ELISA (Untergasser et al., 2006; Iyer et al., 2010; Satoh et al., 2012) and fluorescent RT-PCR (Kim et al., 2009).

\section{Index of cell staining}

Following the standard proposed by Shimizu et al. (Untergasser et al., 2006; von Freyend et al., 2011; Takata et al., 2014), the index for cell staining was calculated as the product of the indices of staining strength and staining percentage. The cell staining strength index was defined as follows: strong staining $=2$; light staining $=1$; and no staining $=0$. The staining percentage index was defined as follows: staining less than $25 \%$ of total cells $=1 ; 25-60 \%$ of total cells $=2$; and $>60 \%$ of total cells $=3$. A strong positive response ( 5 or 6$)$ was denoted as "+++", while positive ( 3 or 4 ), weak positive ( 1 or 2 ), and negative ( 0 ) response were denoted as "++", "+", and "-", respectively.

\section{Statistical analysis}

SPSS v.19.0 was used to analyze all data. Measurements were analyzed by a Student $t$-test, while numbers were analyzed by the chi-square test. $\mathrm{P}<0.05$ was considered to be statistically significant.

\section{RESULTS}

\section{Pathological analysis}

The 62 cases of colorectal cancer included 25 highly differentiated cancers, 21 moderately differentiated tumors, and 16 poorly differentiated cancers. Information on Dukes staging, tumor infiltration, lymph node metastasis, and TNM staging are shown in Table 1.

\section{CK20 expression and correlation with prognosis}

Patients were grouped according to the degree of tumor infiltration and TNM stage, and compared considering the level of CK20 expression. In patients with high levels of infil- 
tration (stage T3 and T4) and distal metastasis (stage III and stage IV), CK20 expression was significantly elevated in both surgical samples and intraperitoneal drainage fluid $(\mathrm{P}<0.05$ compared to T1+T2 or stage I+ stage II patients) as shown in Figure 1A and B. Protein assay by western blot analysis confirmed the high levels of CK20 expression as shown in Figure 1C.

\begin{tabular}{|c|c|c|c|c|c|c|c|}
\hline Dukes staging & N (\%) & Infiltrating stage & N (\%) & TNM staging & N (\%) & Lymph node metastasis & $\mathrm{N}(\%)$ \\
\hline Stage A & $9(14.5 \%)$ & $\mathrm{T} 1$ & $12(19.4 \%)$ & Stage I & $27(43.5 \%)$ & Yes & $30(48.4 \%)$ \\
\hline Stage B & $5(8.1 \%)$ & $\mathrm{T} 2$ & $19(30.6 \%)$ & Stage II & $14(22.6 \%)$ & & \\
\hline Stage C & $37(59.7 \%)$ & T3 & $21(33.9 \%)$ & Stage III & $6(9.7 \%)$ & No & $32(51.6 \%)$ \\
\hline Stage D & $11(17.7 \%)$ & T4 & $10(16.1 \%)$ & Stage IV & $15(24.2 \%)$ & & \\
\hline
\end{tabular}
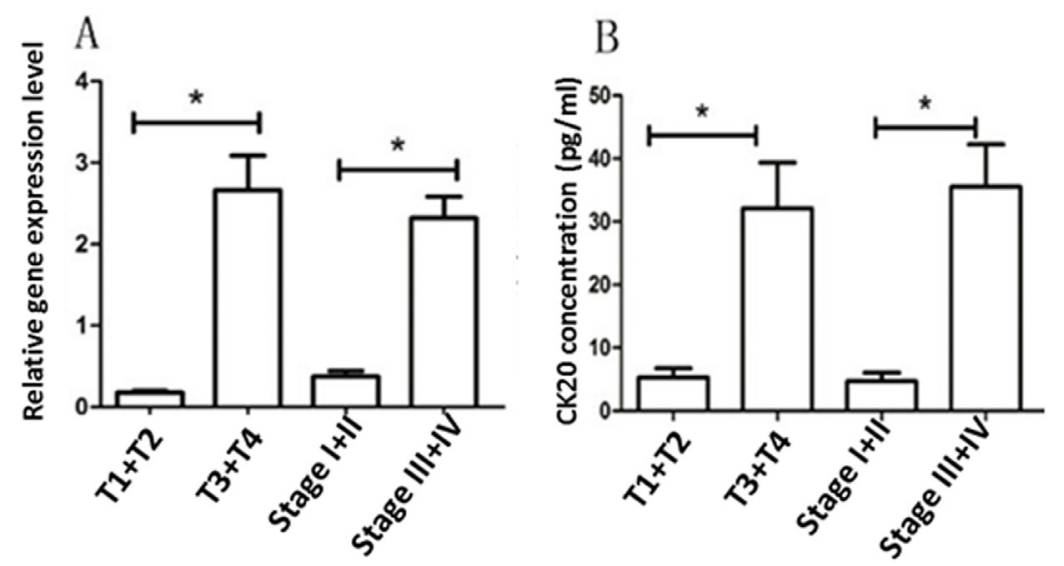

C

T1 T4 Stage I Stage IV

CK20

GADPH

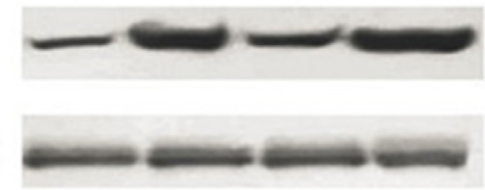

Figure 1. Expression profiles of CK20 in patients with different stages of cancer. A. CK20 mRNA transcripts in colorectal tumor samples. Patients with T3 and T4 tumors had higher levels of CK20 mRNA than patients with T1 and T2 tumors localized more towards the mucosa. When comparing TNM stages, patients with stages III and IV also had higher levels of CK20 mRNA than those with stages I and II. B. CK20 concentrations in intraperitoneal drainage fluid samples. Compared to T1 and T2, those with T3 and T4 had higher levels of CK20 in postoperative intraperitoneal drainage fluids. Patients with TNM stages III and IV also had higher CK20 levels than patients with TNM stages I and II. C. Western blot analysis with colorectal tumor samples. CK20 protein expression was elevated in T4 and stage IV patients compared with that in T1 and stage I patients, respectively. ${ }^{*} \mathrm{P}<0.05$.

\section{Correlation between survival and CK20 expression}

In CK20-positive patients, the rates of postoperative survival at 1, 2, and 3 years were all significantly lower than those in CK20-negative patients (50,37.5, and 12.5\% vs 100, 93.5, 
and $82.6 \%$, respectively; $\mathrm{P}<0.01$ for all comparisons). Considering mRNA levels, patients who were positive for CK20 also had lower postoperative survival rates than those negative for CK20 (survival rates at 1, 2, and 3 years: 93.8, 85.4, and 70.8\% vs 100, 100, and 92.9\%, respectively; $\mathrm{P}<0.05$ for all). Taken together, these results suggest a significant correlation between postoperative prognosis and levels of CK20 in serum and CK20 mRNA levels in lymph nodes (Table 2).

Table 2. Survival of colorectal cancer patients and correlation with CK20 expression.

\begin{tabular}{lcccc}
\hline & $\mathrm{N}$ & 1-year survival (\%) & 2-year survival (\%) & 3-year survival (\%) \\
\hline CK20-positive & 16 & $50(8 / 16)$ & $37.5(6 / 16)$ & $12.5(2 / 16)$ \\
CK20-negative & 46 & $100(46 / 46)$ & $93.5(43 / 46)$ & $82.6(38 / 46)$ \\
$\chi^{2}$ & - & 17.453 & 24.649 & 14.310 \\
P value & - & 0.000 & 0.000 & 0.001 \\
CK20 mRNA-positive & 48 & $93.8(45 / 48)$ & $85.4(41 / 48)$ & $70.8(34 / 48)$ \\
CK20 mRNA-negative & 14 & $100(14 / 14)$ & $100(14 / 14)$ & $92.9(13 / 14)$ \\
$\chi^{2}$ & - & 11.362 & 13.275 & 23.679 \\
P value & - & 0.034 & 0.031 & 0.001 \\
\hline
\end{tabular}

\section{Survival rate and pathological parameters}

Spearman correlation analysis revealed a significant negative relationship between postoperative survival and serum CK20 levels, CK20 mRNA levels in lymph nodes, presence of lymph node metastasis, and grade of infiltration $(\mathrm{r}=-0.843,-0.532,-0.521$ and -0.508 , respectively; $\mathrm{P}<0.05$ for all). However, Dukes stage, TNM stage, or degree of differentiation had no significant correlation with survival $(r=-0.231,-0.236$, and -0.142 , respectively; $P>$ 0.05 for all) as seen in Table 3 .

\begin{tabular}{|c|c|c|}
\hline \multirow[t]{2}{*}{ Pathological parameter } & \multicolumn{2}{|c|}{ Correlation with postoperative survival rate } \\
\hline & $\mathrm{r}$ value & $P$ value \\
\hline Serum CK20 level & -0.843 & 0.000 \\
\hline Lymph node CK20 mRNA level & -0.532 & 0.035 \\
\hline Dukes stage & -0.231 & 0.063 \\
\hline Infiltration grade & -0.508 & 0.045 \\
\hline Lymph node metastasis & -0.521 & 0.036 \\
\hline Differentiation degree & -0.142 & 0.067 \\
\hline TNM stage & -0.236 & 0.052 \\
\hline
\end{tabular}

\section{DISCUSSION}

As no significant symptoms are manifested at the early stages of colorectal cancer, even in the presence of metastasis, it is difficult to diagnose tumors at an early stage and evaluate postoperative prognosis (Sun et al., 2012; Tunca et al., 2013; Jung et al., 2014). In a postoperative follow-up study on 100 patients with colorectal cancer, Li et al. (2010) found significantly higher recurrence rates in patients with lymph node metastasis than in those without metastasis, suggesting a positive relationship between lymph node metastasis and postoperative recurrence of colorectal cancer. A similar result was reported by Ferlay et al. (2010) who found that $28 \%$ of 50 patients with colorectal cancer had lymph node metastasis, indicating 
unfavorable prognosis after surgical resection. Therefore, for effective treatment of colorectal cancer, it is critical to establish a sensitive and specific method to evaluate postoperative prognosis. This study focused on patients with colorectal cancer who underwent surgical resection at our hospital during a 3-year follow-up period. In this cohort, we found that levels of CK20 could effectively predict postoperative prognosis, with a significant correlation between CK20 levels and disease progression.

As fibrous tissue proteins distributed in epithelial cells, the CK protein family participates in the formation of the cytoskeleton and in regulation of cell differentiation and movement (Tunca et al., 2012). Recently, the CK20 protein has received increased interest due to its highly specific expression in both colorectal and gastric cancers (Kim et al., 2009; Sun et al., 2012; Kim et al., 2013; Tunca et al., 2013). In these cells, CK20 protein is highly expressed, with features including irregular modulation, loss of polarity, and diffuse distribution (Šamija et al., 2012). Our study examined tumor tissues at different clinical stages and found higher expression of CK20 with advancement and diffusion of tumor cells. Consistent results were also seen in postoperative intraperitoneal fluid samples (Figure 1C). Our data collectively confirm the role of CK20 as a tumor marker that is expressed in both tumor tissues and peritoneal cavities, whose expression is related to tumor progression. The assay of CK20 levels in intraperitoneal drainage fluids is more feasible in a clinical setting as it is common practice to insert a peritoneal cavity drainage tube after surgical resection (Hainsworth et al., 2012). However, assay of CK20 in intraperitoneal fluids is limited to the acute setting as it is difficult to obtain intraperitoneal fluids in postoperative patients (Lombardo et al., 2011).

Herein, we also examined blood samples from patients during follow-up at 1-3 years after surgery. Expression assays revealed statistically significant correlations between survival at 1,2, and 3 years and serum levels of CK20 and levels of CK20 mRNA in lymph nodes (Table 3), thus suggesting a high predictive value of CK20 in postoperative prognosis. Spearman correlation analysis further revealed a significant negative relationship between survival and serum CK20 levels, lymph node CK20 mRNA levels, presence of lymph node metastasis, and degree of infiltration $(\mathrm{P}<0.05)$, but not Dukes stage, TNM stage, or degree of differentiation $(\mathrm{P}>0.05)$. Therefore, combined assay of serum CK20, lymph node CK20 mRNA, presence of lymph node metastasis, and degree of infiltration is valuable in predicting postoperative prognosis in patients with colorectal cancer.

In summary, both serum CK20 levels and lymph node CK20 mRNA levels significantly correlate with postoperative survival in patients with colorectal cancer. Thus, assay of CK20 expression is valuable in predicting postoperative prognosis. However, only limited confounding factors have been included in this study, and other potential factors such as tumor-induced obstruction or chemotherapy were not assessed. Further studies are therefore required to substantiate the current findings.

\section{Conflicts of interest}

The authors declare no conflict of interest.

\section{ACKNOWLEDGMENTS}

Research supported by funding from the Shanghai Municipal Commission of Health and Family Planning (\#20114005). 


\section{REFERENCES}

Bertz S, Otto W, Denzinger S, Wieland WF, et al. (2014). Combination of CK20 and Ki-67 immunostaining analysis predicts recurrence, progression, and cancer-specific survival in pT1 urothelial bladder cancer. Eur. Urol. 65: 218-226.

Eisenberg-Lerner A, Bialik S, Simon HU and Kimchi A (2009). Life and death partners: apoptosis, autophagy and the cross-talk between them. Cell Death Differ. 16: 966-975.

Ferlay J, Parkin DM and Steliarova-Foucher E (2010). Estimates of Cancer Incidence and Mortality in Europe in 2008. Eur. J. Cancer 46: 765-781.

Hainsworth JD, Schnabel CA, Erlander MG, Haines DW 3rd, et al. (2012). A retrospective study of treatment outcomes in patients with carcinoma of unknown primary site and a colorectal cancer molecular profile. Clin. Colorectal Cancer 11: 112-118.

Iyer P, Zekri AR, Hung CW, Schiefelbein E, et al. (2010). Concordance of DNA methylation pattern in plasma and tumor DNA of Egyptian hepatocellular carcinoma patients. Exp. Mol. Pathol. 88: 107-111.

Jung S, Wu C, Eslami Z, Tanguay S, et al. (2014). The role of immunohistochemistry in the diagnosis of flat urothelial lesions: a study using CK20, CK5/6, P53, Cd138, and Her2/Neu. Ann. Diagn. Pathol. 18: 27-32.

Kim HJ, Lee KW, Kim YJ, Oh DY, et al. (2009). Chemotherapy-induced transient CEA and CA19-9 surges in patients with metastatic or recurrent gastric cancer. Acta Oncol. 48: 385-390.

Kim JH, Rhee YY, Bae JM, Cho NY, et al. (2013). Loss of CDX2/CK20 expression is associated with poorly differentiated carcinoma, the $\mathrm{CpG}$ island methylator phenotype, and adverse prognosis in microsatellite-unstable colorectal cancer. Am. J. Surg. Pathol. 37: 1532-1541.

Li J, Sun RH, Tao KX and Wang G (2010). The CCL21/CCR7 pathway plays a key role in human colon cancer metastasis through regulation of matrix metalloproteinase-9. Dig. Liver Dis. 43: 40-47.

Littman DR and Rudensky AY (2010). Th17 and regulatory T cells in mediating and restraining inflammation. Cell 140: 845-858.

Lombardo Y, Scopelliti A, Cammareri P, Todaro M, et al. (2011). Bone morphogenetic protein 4 induces differentiation of colorectal cancer stem cells and increases their response to chemotherapy in mice. Gastroenterology 140: 297-309.

Montezuma D, Azevedo R, Lopes P, Vieira R, et al. (2013). A panel of four immunohistochemical markers (CK7, CK20, TTF-1, and p63) allows accurate diagnosis of primary and metastatic lung carcinoma on biopsy specimens. Virchows Arch. 463: 749-754.

Pavlidis N and Pentheroudakis G (2012). Cancer of unknown primary site. Lancet 379: 1428-1435.

Šamija I, Lukač J and Kovačević D (2012). Detection of circulating cancer cells in peripheral blood samples obtained before and during surgery for colorectal cancer using cytokeratin-20 as a marker. In: Annual Meeting of the Croatian Immunological Society.

Satoh Y, Mori K, Kitano K, Kitayama J, et al. (2012). Analysis for the combination expression of CK20, FABP1 and MUC2 is sensitive for the prediction of peritoneal recurrence in gastric cancer. Jpn. J. Clin. Oncol. 42: 148-52.

Sun GR, Dong XY, He QS, Qu H, et al. (2012). Expression and clinical significance of CK19 and CK20 expressions in transverse mesocolon biopsies from patients with gastric carcinoma. Cell Biochem. Biophys. 62: 361-364.

Takata A, Kurokawa Y, Fujiwara Y, Nakamura Y, et al. (2014). Prognostic value of CEA and CK20 mRNA in the peritoneal lavage fluid of patients undergoing curative surgery for gastric cancer. World J. Surg. 38: 1107-1111.

Tunca B, Egeli U, Cecener G, Tezcan G, et al. (2012). CK19, CK20, EGFR and HER2 status of circulating tumor cells in patients with breast cancer. Tumori 98: 243-251.

Tunca B, Tezcan G, Cecener G, Egeli U, et al. (2013). Overexpression of CK20, MAP3K8 and EIF5A correlates with poor prognosis in early-onset colorectal cancer patients. J. Cancer Res. Clin. Oncol. 139: 691-702.

Untergasser A, Zedler U, Langenkamp A, Hösel M, et al. (2006). Dendritic cells take up viral antigens but do not support the early steps of hepatitis B virus infection. Hepatology 43: 539-547.

von Freyend MJ, Untergasser A, Arzberger S, Oberwinkler H, et al. (2011). Sequential control of hepatitis B virus in a mouse model of acute, self-resolving hepatitis B. J. Viral Hepat. 18: 216-226.

Zhang W, Xu YC, Guo FJ, Meng Y, et al. (2008). Anti-diabetic effects of cinnamaldehyde and berberine and their impacts on retinol-binding protein 4 expression in rats with type 2 diabetes mellitus. Chin. Med. J. 121: 2124-2128. 